\title{
Polibotánica
}

ISSN electrónico: 2395-9525

POLIBETÁNICA polibotanica@gmail.com Instituto Politécnico Nacional México http:www.polibotanica.mx

\section{MORFOLOGÍA Y GERMINACIÓN DE Chamaedorea glaucifolia (ARECACEAE), ESPECIE EN PELIGRO Y ENDÉMICA DE MÉXICO}

\section{MORPHOLOGY AND GERMINATION OF Chamaedorea glaucifolia (ARECACEAE), ENDANGERED AND ENDEMIC SPECIES OF MEXICO}

Orantes-García, C.; O. Farrera-Sarmiento, R. Gutiérrez-González, R. A. Moreno-Moreno, E. R. Garrido-Ramírez, y S. J. Reyes-Zambrano MORFOLOGÍA Y GERMINACIÓN DE Chamaedorea glaucifolia (ARECACEAE), ESPECIE EN PELIGRO Y ENDÉMICA DE MÉXICO.

MORPHOLOGY AND GERMINATION OF Chamaedorea glaucifolia (ARECACEAE), ENDANGERED AND ENDEMIC SPECIES OF MEXICO.

POLIBeTÁNICA

Instituto Politécnico Nacional
Núm. 47: 77-87 México. Enero 2019

DOI: $10.18387 /$ polibotanica.47.6 


\section{MORFOLOGÍA Y GERMINACIÓN DE Chamaedorea glaucifolia (ARECACEAE), ESPECIE EN PELIGRO Y ENDÉMICA DE MÉXICO}

\section{MORPHOLOGY AND GERMINATION OF Chamaedorea glaucifolia (ARECACEAE), ENDANGERED AND ENDEMIC SPECIES OF MEXICO}

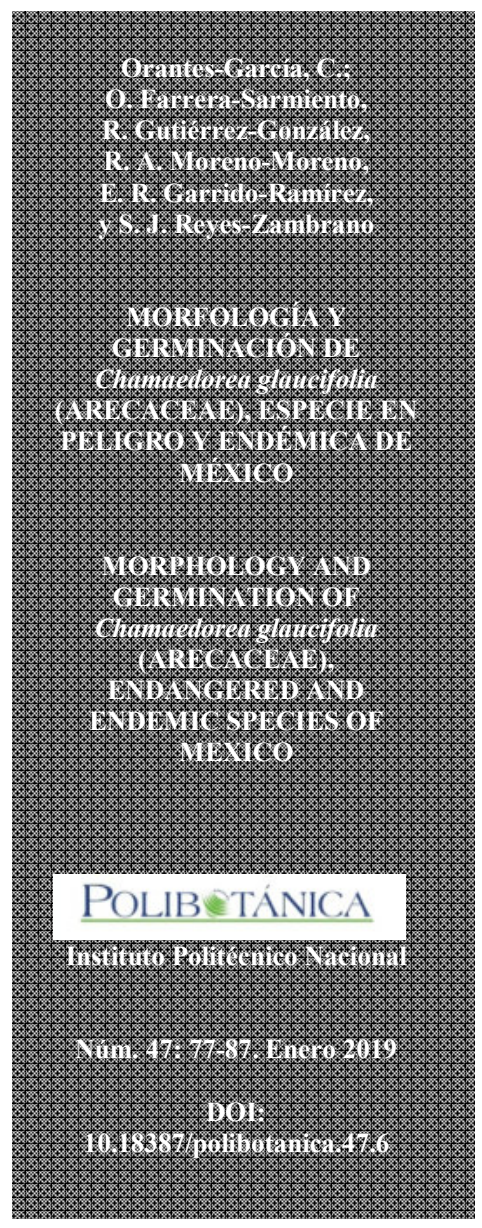

RESUMEN: Chamaedorea glaucifolia H. Wendl, es una especie de la familia Arecaceae, considerada endémica del sureste de México y catalogada en peligro según la Nom-059-Semarnat-2010, dada la importancia ornamental de esta palma y la carencia de estudios enfocados a su germinación, almacenamiento y morfometría, el presente trabajo tuvo como objetivo evaluar el efecto del almacenamiento sobre la viabilidad y germinación de la semilla; así como determinar características morfométricas de la infrutescencia, fruto y semilla. A 100 frutos y semillas tomados al azar se les determinó peso, longitud y diámetro de manera individual, para el efecto del periodo de almacenamiento (30, 60, 90 y 120 días), se evaluó la viabilidad mediante la prueba de tetrazolio y la germinación con la siembra de semilla, en un diseño experimental completamente aleatorio con cuatro repeticiones de 25 semillas cada una, haciendo un total de 100 semillas por tratamiento. En promedio las infrutescencias contienen 1113.75 frutos $(\mathrm{N}=5)$. El fruto presenta $0.85 \pm 0.52 \mathrm{~cm}(\mathrm{~N}=100)$ de longitud, $0.70 \pm$ 
$0.35 \mathrm{~cm}(\mathrm{~N}=100)$ de diámetro y $0.25 \pm 0.028 \mathrm{~g}(\mathrm{~N}=100)$ de peso. Cada fruto contiene una semilla globosa, con longitud de $0.62 \pm 0.21 \mathrm{~cm}(\mathrm{~N}=100)$, diámetro de $0.52 \pm 0.28 \mathrm{~cm}(\mathrm{~N}=$ 100) y $0.11 \pm 0.025 \mathrm{~g}(\mathrm{~N}=100)$ de peso, un kilogramo contiene en promedio 8500 semillas. Las semillas presentaron $100 \%$ de viabilidad a los 30 días de almacenamiento, esto desciende hasta $65 \%$ a los 120 días, la capacidad germinativa de las semillas también es afectada por el periodo de almacenamiento. La germinación es hipogea, la radícula en semillas con 120 días de almacenamiento, empieza a emerger a los 49 días después de la siembra (dds). Los estudios sobre morfometría, viabilidad y germinación permiten obtener información fundamental para la propagación de especies en riesgo como C. glaucifolia.

Palabras claves: Propagación, almacenamiento, vialidad, palmas, Chiapas.

\begin{abstract}
Chamaedorea glaucifolia H. Wendl is a species of the family Arecaceae, considered endemic to the southeast of Mexico and classified in danger according to the Nom059-Semarnat-2010, given the ornamental importance of this palm and the lack of studies focused on its germination, storage and morphometry, the present work had as objective to determine the morphometric characteristics of the infructescence, fruit and seed, as well as to study the germinative process and the effect of storage on the viability and germinative capacity. To 100 fruits and seeds taken at random were individually determined weight, length and diameter, for the effect of the storage period (30, 60, 90 and 120 days), viability was assessed by tetrazolium test and germination with seed sowing, in a completely random experimental design with four replicates of 25 seeds each, making a total of 100 seeds per treatment. On average the infructescences contain 1113.75 fruits $(\mathrm{N}=5)$. The fruit presented $0.85 \pm 0.52 \mathrm{~cm}(\mathrm{~N}=100)$ in length, $0.70 \pm 0.35 \mathrm{~cm}(\mathrm{~N}=100)$ in diameter and $0.25 \pm 0.028 \mathrm{~g}$ $(\mathrm{N}=100)$ in weight. Each fruit contains a globose seed with a length of $0.62 \pm 0.21 \mathrm{~cm}(\mathrm{~N}=$ $100)$, a diameter of $0.52 \pm 0.28 \mathrm{~cm}(\mathrm{~N}=100)$ and $0.11 \pm 0.025 \mathrm{~g}(\mathrm{~N}=100)$ weight, one kilogram contains on average 8500 seeds. The seeds presented $100 \%$ viability at 30 days of storage, this drops to $65 \%$ at 120 days, the germination capacity of the seeds is also affected by the storage period. Germination is hypogeal, the radicle in seeds with 120 days of storage, begins to emerge at 49 days after sowing (das). Studies on morphometry, viability and germination allow obtaining fundamental information for the propagation of endangered species such as C. glaucifolia, which urgently needs conservation and management strategies to reduce the impact on wild populations.
\end{abstract}

Keywords: Propagation, storage, viability, palms, Chiapas.

\title{
INTRODUCCIÓN
}

La familia Arecaceae (palmas) se distingue por su gran abundancia y su alta diversidad morfológica y funcional (Henderson et al., 1995). Hay aproximadamente 200 géneros con 1500 especies de palmas en el mundo, de los cuales 67 géneros con 550 especies se encuentran naturalmente en América tropical (Grayum, 2003). En México existen aproximadamente 22 géneros y 86 a 100 especies, de los cuales alrededor de 50 especies son del género Chamaedorea (Quero, 1994), entre estas se encuentran especies endémicas, regional o localmente, como C. alternans, C. cataractarum, C. glaucifolia, C. hooperiana, C. klotzchiana, C. oreophila, C. queroana, C. schiediana y las palmas de hojas bífidas C. metallica, C. rigida, C. stolonifera. La mayor riqueza y diversidad de este género se encuentran en el sur de México, entre los Estados de Chiapas, Oaxaca y Veracruz (Gámez-Pastrana et al., 2016; Henderson et al., 1995; Quero, 1994).

Chamaedorea, tiene importancia económica tanto a nivel mundial como nacional, no solamente por su participación en el mercado de materias primas y su consecuente contribución económica (Hodel, 1992), sino también porque son parte de la vida cotidiana de las comunidades rurales. En México, la palma camedor, es un recurso forestal no maderable que ha sido aprovechado en 
diversas formas; la de mayor importancia económica es la comercialización de su follaje con fines de ornato (López-Paniagua et al., 2005). De esta planta también se puede obtener una variedad de productos y subproductos como material para las viviendas, vestido, combustibles, fibras, alimentos, medicinas y artesanías, frecuentemente la explotación desmedida de este recurso vegetal implica un riesgo elevado de extinción (Toledo et al.,1989). El aprovechamiento irracional, ha causado una alarmante disminución en las poblaciones naturales de la palma camedor (Granados-Sánchez et al., 2004). Aunado a lo anterior, el avance de la agricultura y ganadería en su hábitat natural, además de la explotación de los bosques y selvas para extracción de maderas, han provocado la pérdida de la biodiversidad y el cambio en el uso del suelo en las regiones tropicales (Pérez et al., 2007; Villafuerte et al.,1997). Este hecho ha obligado a las autoridades mexicanas incluir en la lista de la Nom-059-Semarnat-2010 a 38 especies de Chamaedorea, entre las cuales se encuentra $C$. glaucifolia con distribución endémica y catalogada como especie en peligro (Espejo, 2001).

En Chiapas, Chamaedorea glaucifolia (palma camedor despeinado), se distribuye en la Depresión Central, en las montañas de la parte norte del estado, en la Reserva de la Biosfera Selva el Ocote, principalmente en los bosques tropicales de hoja caduca en altitudes que van desde 500 a 1000 m; en el Cañón del Sumidero puede encontrarse a $1200 \mathrm{~m}$ de altitud (EspinosaJiménez et al., 2011; Henderson et al., 1995; Hodel, 1992). Esta especie es extremadamente rara y se encuentra en poblaciones pequeñas con pocos individuos (Hodel, 1992).

También es notoria la poca o nula investigación referente a aspectos de germinación, viabilidad y características sobre la morfología de Chamaedorea glaucifolia. Cabe señalar, que los estudios de germinación y viabilidad de las semillas son de suma importancia para poder establecer programas de conservación y cultivo (Forcella et al., 2000; Allison, 2002; Abud et al., 2010; Miransari y Smith, 2014). El almacenamiento, puede afectar la viabilidad (capacidad de vida) y la capacidad de germinación de la semilla; provocando reducción en el potencial de producción de cultivos, pérdida de calidad debido al envejecimiento de la semilla, puede causar un retraso en el establecimiento en campo, anomalías en las plántulas o incluso la falla de la emergencia (Nautiyal et al., 2003; Kandari et al., 2012; Sharma et al., 2014).

Por lo que este trabajo pretende evaluar el efecto del almacenamiento sobre la viabilidad y germinación de la semilla de Chamaedorea glaucifolia; así como determinar características morfométricas de la infrutescencia, fruto y semilla, con la finalidad de aportar información básica sobre la especie.

\section{MATERIAL y MÉTODOS}

La recolecta de los frutos se llevó acabo en enero 2013 en la Reserva Estatal denominada Zona Sujeta a Conservación Ecológica La Pera, localizada en la porción central del municipio de Berriozábal, Chiapas, México, a 950 m s.n.m., $16^{\circ} 44^{\prime} 24^{\prime \prime}$ N y $92^{\circ} 58^{\prime} 12^{\prime}$ ' W, dentro del ecosistema de Bosque tropical subcaducifolio. Se eligieron cinco infrutescencias maduras y completas de cinco plantas ubicadas en un cuadrante de 1 ha (una infrutescencia por planta), se cortaron los frutos y se transportaron al laboratorio en bolsas de papel.

Los frutos fueron separados manualmente, se realizó la remoción del exocarpio, mesocarpio y endocarpio de forma manual, las semillas se dejaron secar a la sombra durante cuatro días y se almacenaron en un recipiente hermético de plástico transparente, colocándolos en una alacena de madera libre de humedad y a temperatura ambiente $\left(25 \pm 1^{\circ} \mathrm{C}\right)$. Se determinó el número de frutos por infrutescencia y número de semillas por kilogramo. En un lote de 1000 frutos se tomaron 100 al azar, de manera individual a los frutos y semillas se les determinó el peso, longitud y diámetro.

El efecto del periodo de almacenamiento se evaluó mediante dos pruebas: 


\section{Viabilidad}

El propósito de esta prueba fue comparar la viabilidad (capacidad de vida) de las semillas después de 30, 60, 90 y 120 días de almacenamiento. Para realizar la prueba se formaron cuatro grupos de semillas, uno por cada periodo de almacenamiento. Cada grupo fue tratado como se describe a continuación: se humedecieron tres lotes con 100 semillas durante $24 \mathrm{~h}$ para facilitar un corte longitudinal en la testa y exponer el embrión a la solución de tinción $(0.5 \%$ cloruro 2 , 3,5 trifenil-2H tetrazolio). Las semillas fueron colocadas en cajas Petri y se envolvieron con papel aluminio para limitar su exposición a la luz. La incubación se realizó en un ambiente libre de humedad y luz, a temperatura ambiente $\left(25^{\circ} \mathrm{C}\right)$ durante $24 \mathrm{~h}$ (International Seed Testing Association, 2005). Cada semilla se diseccionó y los embriones se observaron con un microscopio estereoscópico marca Carl Zeizz ${ }^{\circledR}$, para contar el número de semillas totalmente teñidas. Los datos correspondientes al número de semillas viables (según la International Seed Testing Association 2005) fueron transformados a arcoseno raíz cuadrada para realizar un análisis de varianza (ANOVA) con el apoyo del paquete estadístico SPSS v.20 (SAS, 2011), de acuerdo a un diseño completamente aleatorizado (Montgomery, 2002).

\section{Germinación}

El efecto del periodo de almacenamiento se evaluó con la siembra de las semillas almacenadas durante 30, 60, 90 y 120 días. Se utilizaron un total de 100 semillas por tratamiento, se sembraron en charolas de germinación con tubetes monoblock inyectadas de polietileno tipo forestal a una profundidad de $2 \mathrm{~cm}$ con el micrópilo hacia abajo en sustrato a base de peatmoss, agrolita y vermiculita $(3: 1: 1 \mathrm{v} / \mathrm{v})$, en un umbráculo con $70 \%$ de sombra, a una temperatura promedio de $25^{\circ} \mathrm{C}$ y $70 \%$ de humedad relativa aproximadamente. El periodo de observación fue de 220 días, con registros cada 10 días y riego a capacidad de campo realizados cada tres días. Se consideraron germinadas las semillas cuando presentaron emergencia sobre el sustrato (Hartmann, H.T. y Kester, 1994). Se aplicó un diseño experimental completamente aleatorio con cuatro repeticiones de 25 semillas cada una (Montgomery, 2002). Se evaluó el porcentaje de germinación final $(\% \mathrm{G})$, proporción de semillas capaces de germinar en condiciones óptimas o en una condición determinada (Bewley y Black, 1994); germinación acumulada, que muestra la forma en que se incrementa la germinación y el tiempo (días) de inicio de la germinación; tiempo promedio de germinación, que son los días requeridos para la emergencia sobre el sustrato (González y Orozco, 1996):

a) Porcentaje de germinación final $(\% \mathrm{G})$.

$$
\% \mathrm{G}=\frac{n i}{N} \times 100
$$

Dónde: $\mathrm{N}=$ Total de semillas sembradas, $\mathrm{ni}=$ número total de semillas germinadas.

b) Germinación acumulada (GA).

$$
\mathrm{GA}=\frac{\% \mathrm{n} 1+\% \mathrm{n} 2+\% \mathrm{n} 3+\% \mathrm{nx}}{\mathrm{nx}}
$$

Dónde: $\% \mathrm{n} 1=$ porcentaje de semillas germinadas en el tiempo $1, \% \mathrm{n} 2=$ porcentaje de semillas germinadas en el tiempo $2, \% \mathrm{n} 3=$ porcentaje de semillas germinadas en el tiempo $3 \mathrm{y} \mathrm{nx}=$ tiempo en que se presentó la germinación.

c) Tiempo promedio de germinación (T). 


$$
\mathrm{T}=\frac{\sum(\text { niti) }}{\sum \mathrm{ni}}
$$

Dónde: $\mathrm{ni}$ = número de semillas germinadas en el día $\mathrm{i}, \mathrm{y}$ ti = tiempo de germinación desde la siembra hasta la germinación de la última semilla.

Se realizó la descripción del proceso germinativo de las semillas; determinando tipo de germinación, emergencia de la radicula, epicotilo, raíces y formación de hojas, debido al tiempo del experimento, esto se llevó a cabo en semillas con 120 días de almacenamiento.

Los datos de germinación fueron transformados a arco seno raíz cuadrada, se realizó un análisis de varianza (ANOVA), a fin de verificar el efecto del período de almacenamiento sobre la germinación de las semillas, se obtuvieron los coeficientes de regresión polinómica. Los análisis estadísticos se realizaron utilizando el paquete estadístico SPSS v.20 (SAS, 2011).

\section{RESUlTADOS}

\section{Descripción del fruto y semilla}

En el área de recolecta, se pueden encontrar plantas de C. glaucifolia con hasta cinco infrutescencias (racimos), las cuales en promedio tienen 1113.75 frutos $(\mathrm{N}=5)$ (fig. 1A) y la producción es anual. Los valores promedio por tamaño de fruto fueron de $0.85 \pm 0.52 \mathrm{~cm}(\mathrm{~N}=$ $100)$ de longitud, $0.70 \pm 0.35 \mathrm{~cm}(\mathrm{~N}=100)$ de diámetro y $0.25 \pm 0.028 \mathrm{~g}$ de peso $(\mathrm{N}=100)$, un kilogramo contiene en promedio 8500 semillas $(\mathrm{N}=5)$.

Cada fruto contiene una semilla de forma globosa, con longitud promedio de $0.62 \pm 0.21 \mathrm{~cm}(\mathrm{~N}$ $=100)$, diámetro de $0.52 \pm 0.28 \mathrm{~cm}(\mathrm{~N}=100)$ y $0.11 \pm 0.025 \mathrm{~g}$ de peso $(\mathrm{N}=100)$ (fig. $1 \mathrm{~B})$. La semilla presenta una testa delgada papirácea de color café, micrópilo conspicuo, hilium basal, el rafe se distingue fácilmente (fig. 1C).

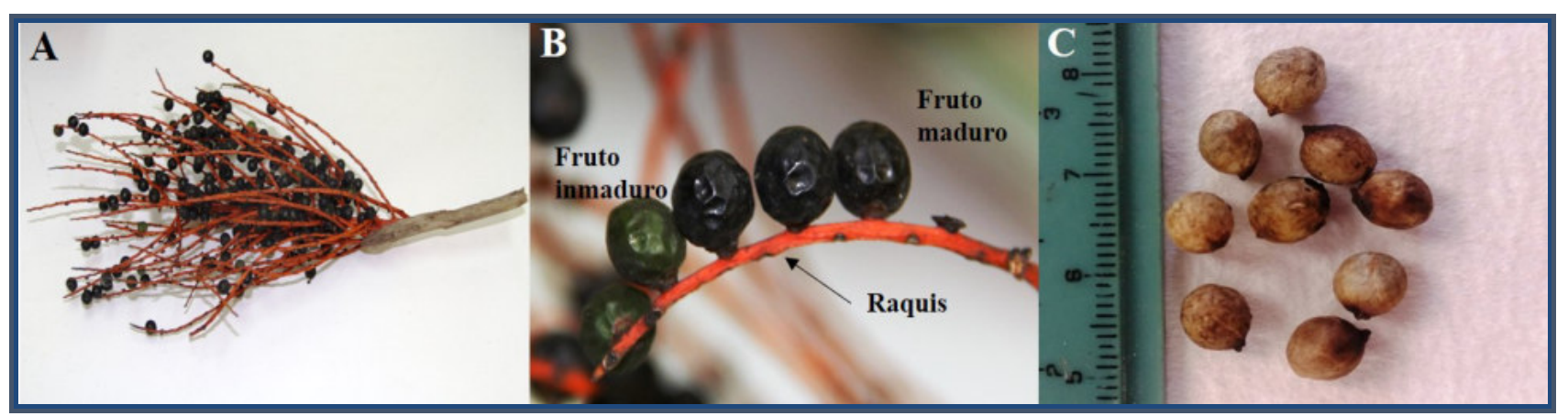

Fig. 1. A. Infrutescencia racimosas de Chamaedorea glaucifolia. B. Fruto pseudodrupa, globoso, negro cuando está maduro, epicarpio liso, mesocarpio escasamente carnoso y endocarpio delgado fibroso. C. Semilla globosa ovoide. (Henderson et al., 1995; Martínez et al., 2007)

La testa envuelve un endospermo compacto, vidrioso, donde se encuentra un embrión rudimentario de $\leq 0,35 \mathrm{~cm}$ de largo, de forma cilíndrica, amarillo claro, consistencia suave, posición basal cerca del micrópilo, el epicótilo y la radícula no presentan muchas diferencias, excepto que la radícula en la parte inferior tiende a ser más ancha (fig. 2). 


\section{Viabilidad y germinación}

A los 30 días de almacenamiento las semillas presentaron $100 \%$ de viabilidad y $73 \%$ de germinación final (cuadro 1). Se encontraron diferencias significativas $(p \leq 0,01)$ entre los tiempos de almacenamiento, lo cual confirma que el período de almacenamiento provocó una disminución en el porcentaje de viabilidad y germinación de las semillas, se observó que las semillas con 120 días de almacenamiento obtuvieron $65 \%$ de viabilidad y $45 \%$ de germinación.

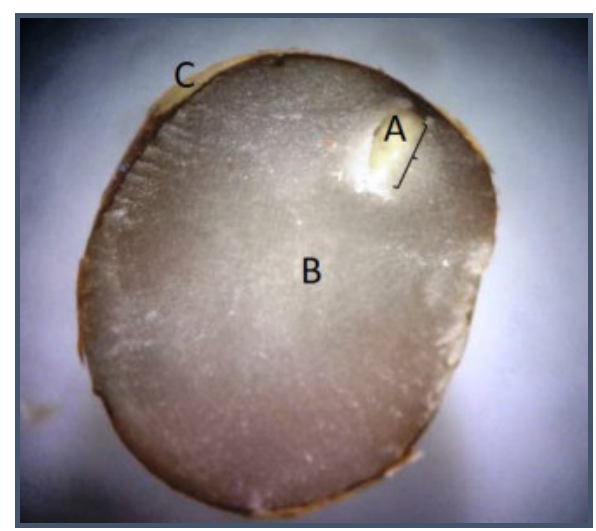

Fig. 2. Corte longitudinal de la semilla de Chamaedorea glaucifolia, A. Embrión de $\leq 0,35 \mathrm{~cm}$ de largo, próximo al micrópilo. B. Endospermo compacto vidrioso con presencia de aceite. C. Testa delgada papirácea de color café, que envuelve al embrión.

Cuadro 1. Comparación de los promedios de los datos de germinación de Chamaedorea glaucifolia, en los diferentes periodos de almacenamiento. Porcentaje de viabilidad $(\% \mathrm{~V})$, Porcentaje final de germinación $(\% \mathrm{G})$,

Germinación acumulada (GA), Tiempo promedio de germinación (T).

\begin{tabular}{ccccc}
\hline $\begin{array}{c}\text { Tiempo de } \\
\text { almacenamiento } \\
\text { (días) }\end{array}$ & $\% \mathbf{~ V}$ & $\% \mathbf{~ G}$ & GA (días) & T (días) \\
\hline 30 & $100 \pm 2.3^{\mathrm{a}^{\mathrm{a}}}$ & $73 \pm 21.5^{\mathrm{a}}$ & $18.25 \pm 5.37^{\mathrm{a}}$ & $149.10 \pm 3.54^{\mathrm{a}}$ \\
60 & $85 \pm 4.5^{\mathrm{b}}$ & $66.5 \pm 16.6^{\mathrm{b}}$ & $15.75 \pm 4.27^{\mathrm{b}}$ & $100.25 \pm 2.86^{\mathrm{bc}}$ \\
90 & $70 \pm 3.0^{\mathrm{c}}$ & $49 \pm 15.7^{\mathrm{c}}$ & $12.25 \pm 3.94^{\mathrm{c}}$ & $100.40 \pm 10.7^{\mathrm{bc}}$ \\
120 & $65 \pm 5.3^{\mathrm{d}}$ & $45 \pm 9.4^{\mathrm{d}}$ & $11.25 \pm 2.36^{\mathrm{d}}$ & $75.94 \pm 3.41^{\mathrm{c}}$ \\
\hline
\end{tabular}

Tamaño de población $=100$ semillas, $*$ Promedio \pm desviación estándar.

Las semillas con 30 días de almacenamiento inician la emergencia a los 92 días después de la siembra (dds) y finalizan a los 192 dds, se observó que conforme aumenta el periodo de almacenamiento las semillas requieren de menos tiempo para emerger. 


\section{Descripción del proceso germinativo de Chamaedorea glaucifolia}

La germinación es hipogea, la radícula empieza a emerger de la semilla a los 49 días después de la siembra (dds), tiene una forma cónica, ligeramente arqueada con la punta redondeada, longitud de $0.3 \mathrm{~cm}$. A los 60 dds se puede observar el epicótilo, el cual tiene una forma cilíndrica y una longitud de $0.3 \mathrm{~cm}$, mientras que la radícula ahora mide $0.6 \mathrm{~cm}$. A los $77 \mathrm{dds}$ el epicótilo y la radícula siguen creciendo; la radícula tiene una longitud de $1.5 \mathrm{~cm}$, mientras que el epicótilo mide $1.2 \mathrm{~cm}$. A los $97 \mathrm{dds}$ ya se pueden observar cambios significativos en la estructura de la plántula; la raíz se ha alargado hasta medir $11 \mathrm{~cm}$ de longitud, además las raíces laterales han comenzado a emerger, por su parte la vaina foliar mide ahora $3 \mathrm{~cm}$ y es posible observar el inicio de la formación de la primera hoja. La raíz primaria ha alcanzado una longitud de $15 \mathrm{~cm}$ a los $131 \mathrm{dds}$, siendo esta su longitud máxima dentro del tubete, ya que este mide $15 \mathrm{~cm}$, además se puede observar una considerable cantidad de raíces secundarias, por su parte la vaina foliar mide $5 \mathrm{~cm}$ y ha desarrollado su primera hoja, la cual tiene la forma característica de cola de pescado, es de color verde y muy flexible. La primera hoja compuesta se puede observar a los $272 \mathrm{dds}$; la raíz primaria sigue midiendo $15 \mathrm{~cm}$, pero las raíces secundarías se han extendido a lo ancho por todo el tubete, por su parte la vaina foliar mide 15 $\mathrm{cm}$ de longitud y presenta su primera hoja compuesta, la cual es de un color verde, ligeramente más intenso que su hoja primaria (fig. 3).

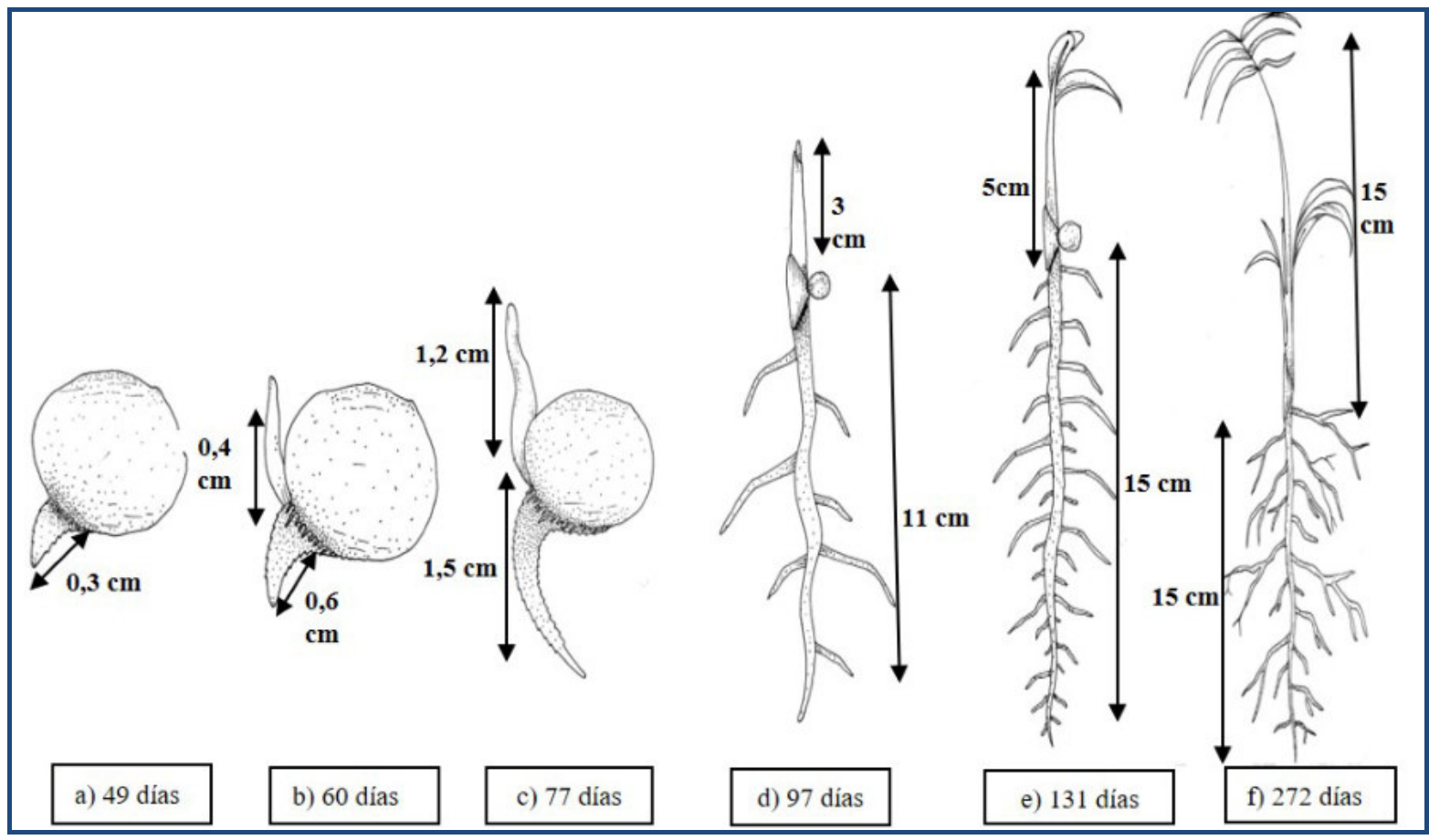

Fig. 3. Proceso germinativo de Chamaedorea glaucifolia, durante los primeros 272 días después de la siembra. Datos tomados en semillas con 120 días de almacenamiento.

\section{DISCUSIÓN}

En este estudio se reporta por primera vez las características morfométricas de la infrutescencia, fruto y semilla de C. glaucifolia, las cuales presentaban en promedio 1113.75 frutos, tallos de aproximadamente $2 \mathrm{~m}$ de altura (en ocasiones mayor a $5 \mathrm{~m}$ ), 2-3.5 $\mathrm{cm}$ de diámetro, nudos 
prominentes, entrenudos 25-35 cm de largo. De 3-5 hojas pinnadas, erectas, haciendo alusión a plumas. Inflorescencia intrafoliar entre vainas de las hojas viejas, erecto, solitario, con pedúnculo largo. Flores estaminadas, globosas, amarillas, ligeramente aromáticas. Frutos globosos, negro, 0.7-1 cm de diámetro. El número de frutos es variable dependiendo de la especie, por ejemplo, en Chamaedorea ernesti-augustii varían de 40 a 50 , mientras que en $C$. elegans superan los 500; son de forma globosa, color ligeramente anaranjado en el proceso de maduración y negros cuando están maduros, su tamaño máximo alcanza los $10 \mathrm{~mm}$ de diámetro; presentan semillas globosas de 8 a $10 \mathrm{~cm}$ de diámetro (Zamora-Martinez et al., 2011)

El conocimiento del proceso de germinación es importante ya que permite, explicar en parte, el ciclo de vida de la especie. Se reconoce que la germinación de semillas y la supervivencia de las plántulas son una parte crucial del ciclo de vida de las plantas y de su dinámica poblacional (Orozco et al., 2003).

En muchas especies el tiempo de almacenamiento influye en la germinación (Baskin y Baskin, 2001). Diversas fuentes corroboran el efecto negativo del tiempo de almacenamiento en semillas, tal como señalan (Mora et al., 2003; Rojas et al., 2000) para el género Chamaedorea. Dicha declinación en la germinación obedece a la reducción del contenido de humedad en la semilla, conforme pasan más tiempo en almacenamiento (Carpenter y Ostmark, 1994; Vázquez et al., 1999; Rodríguez et al., 2000).

La pérdida de viabilidad en semillas bajo condiciones de almacenamiento ha sido reportada para otras especies de palmas y se ha sugerido que la desecación del embrión es la causa de dicha pérdida (Orozco et al., 2003) Aún bajo condiciones óptimas conforme aumenta el tiempo de almacenamiento, la viabilidad de la semilla desciende paulatinamente (Hartmann y Kester, 1994). La disminución de la viabilidad puede deberse también la falta de integridad de las membranas celulares de las semillas, por disminución de fosfolípidos, carbohidratos y proteínas, lo que provoca que la estructura y funcionamiento de las semillas se modifique, y se generen cambios en la permeabilidad, pérdida de fluidez e integridad (Melo y Cuamatzi, 2008).

\section{CONCLUSIÓN}

Se pudo observar como el tiempo de almacenamiento afecta la germinación y viabilidad de las semillas de C. glaucifolia. Si se desea cultivar o almacenar las semillas de C. glaucifolia sería conveniente no tenerlas almacenadas más de 30 días, en un periodo de almacenamiento mayor (60-120 días) el porcentaje de germinación y viabilidad de estas semillas disminuye. La realización de estudios básicos sobre la germinación y viabilidad de C. glaucifolia representa una estrategia importante para el manejo y conservación de esta especie, que se encuentra reportada en peligro de extinción.

\section{LITERATURA CITADA}

Abud, H., Reis, R., Innecco, R., y Bezzerra, A. (2010). Emergence and development of seedlings of safflower depending on seed size. Rev. Ciên. Agron, 41, 95-99.

Allison, V. J. (2002). Nutrients, arbuscular mycorrhizas and competition interact to influence seed production and germination success in Achillea millefolium. Funct. Ecol., 16, 742-749.

Baskin, C.C., y Baskin, J. M. (2001). Seeds: ecology, biogeography and evolution of dormancy and germination. (Academic Press., Ed.) (2nd ed.). San Diego, CA.

Bewley, J. y Black, M. (1994). Seeds, physiology of development and germination. (P. Press, Ed.). New York.

Carpenter, W.J., Y., \& Ostmark, E. R. (1994). Temperature and Desiccation Affect the Germination of Chamaedorea Palm Seed. Hortscience, 107, 183-186. 
Espejo, S. A. (2001). El endemismo en las Liliopsida mexicanas. Acta Botánica Mexicana, 100, $195-257$.

Espinosa-Jiménez, J.A., Pérez-Farrera, M.Á. y Martínez-Camilo, R. (2011). Inventario florístico del Parque Nacional Cañón del Sumidero, Chiapas, México. Boletín de La Sociedad Botánica de México., 29, 37-82.

Forcella, F., Arnold, R., Sánchez, R., y Ghersa, C. (2000). Modeling seedling emergence. Field. Crops. Res., 67, 123-139.

Gámez-Pastrana, M.R., García-Castillo, M.A., Galindo-Tovar, M.E. y Gheno-Heredia, Y. A. (2016). Diversidad y distribución del género Chamaedorea (Arecaceae) en México. Agroproductividad, 9(6), 10-19.

González, Z., y Orozco, S. (1996). Métodos de análisis de datos en la germinación de semillas, un ejemplo: Manfreda Brachystachya. Boletín de La Sociedad Botánica de México, 58, $15-30$.

Granados-Sánchez, D., Hernández G.M.A., López-Ríos, G.F. y Santiago, L. M. (2004). El cultivo de palma camedor (Chamaedorea sp.) en sistemas agroforestales de Cuichapa, Veracruz. Revista Fitotecnia Mexicana, 27(3), 233-241.

Grayum, M. H. (2003). Arecaceae. In N. Hammel, B.E., Grayum, M.H, Herrera, C., Zamora (Ed.), Manual de plantas de Costa Rica (pp. 201-293). Missouri Botanical Garden.

Hartmann, H.T. y Kester, D. E. (1994). Propagación de Plantas y Principios Básicos. (CECSA, Ed.). México, D. F.

Henderson, A., Galeano, G. y Bernal, R. (1995). Field Guide to the Palms of the Americas. (P. U. Press, Ed.). Princeton New Jersey.

Hodel, D. R. (1992). Chamaedorea Palms: The species and their Cultivation. (A. Press, Ed.). Lawrence, Kansas.

Kandari, L.S., Rao, K.S., Payal, K.C., Maikhuri R.K., Chandra, A., y Van Staden, J. (2012). Conservation of aromatic medicinal plant Rheum emodi through improvedseed germination. Seed Sci. Technol., 40, 95-101.

López-Paniagua, J. E., Bolaños-Mendez, M., De los Santos-Espinoza, y Martinez-Alvarado, N. (2005). Manual para el manejo y cultivo de palma comedor. (A. C. Grupo Mesófilo, Ed.). México. Retrieved from http://www.grupomesofilo.org/pdf/manuales/ manual_palma_camedor.pdf

Melo, R. V. y Cuamatzi, T. (2008). Bioquímica de los procesos metabólicos. (Reverté, Ed.) (2nd ed.). México.

Miransari, M., y Smith, D. L. (2014). Plant hormones and seed germination. Environ. Exp. Bot., 99(110-121).

Montgomery, D. (2002). Diseño y análisis de experimentos (2nd ed.). Mexico D.F.: Limusa, S.A. de C.V.

Mora, A., Rodríguez J., Peña L. y Ramírez, L. (2003). Respuesta de Chamaedorea elegans Mart. a tratamientos pregerminativos. Revista Chapingo Serie Horticultura, 9, 135-141.

Nautiyal, B.P., Prakash, V., Maithan, U.C., Chauhan, R.S., Purohit, H., y Nautiyal, M. C. (2003). Germinability: productivity and economic viability of Rheum emodi Wall. ex Meissn. cultivated at lower altitude. Curr. Sci., 84(143-148).

Orozco, S.A., Batis, A., Rojas, A.M., y Mendoza, A. (2003). Seed biology of palms: a review. Palms, 47, 79-94.

Pérez, F.M.A., Vovides, A.P., Iglesias C., Martínez, M.N. y Martínez, C. R. (2007). New reports of endangered Chamaedorea species (Arecaceae) from southeastern Mexico, with notes on conservation status, habitat, and distribution. Rhodora, 109(938), 187196.

Quero, H. J. (1994). Flora de Veracruz: Palmae. (pp. 31-35). Veracruz, México: Instituto de ecología.

Rodríguez, M.C. Orozco-Segovia, A., Sánchez-Coronado M.E. y Vázquez-Yanes, C. (2000). Seed germination of six mature neotropical rain forest species in response to dehydration. Tree Physiology, 20(10), 693-699. https://doi.org/10.1093/ treephys/20.10.693 
Recibido:

$14 /$ mayo/2018

Aceptado:

10/diciembre/2018
Rojas, A.M., Jurado E., Sánchez R.G., Trejo H.L. y Leal, R. F. (2000). Rapid viability loss in seeds of palmilla (Chamaedorea radicalis Mart.) from El Cielo Biosphere Reserve. The Southwestern Naturalist, 45(3), 373-375.

SAS. (2011). SPSS v. 20 Statistics and graphics guide. Statistical discovery software. Institute Cary. N.C.

Sharma, R.K., Sharma, S., y Sharma, S. S. (2014). The long-term ambient storage-induced alterations in seed viability, germination and seedling vigour of Saussurea costus, a critically endangered medicinal herb of North West Himalaya. J. Appl. Res. Med. Aromat. Plants, 1(92-97).

Toledo, V.M., Carabias, J., Toledo, C., y González-Pacheco, C. (1989). La Producción Rural en México. México, D.F.: Fundación Universo Veintiuno.

Vázquez, Y.C., Orozco S.A. y Sánchez, C. M. E. (1999). Recalcitrance among the seeds of the woody plants growing at the Northern limit of the tropical rain forest in the American continent. In Proceedings of the IUFRO Seed Symposium. Kuala Lumpur, Malasya.

Villafuerte, D., García, M. y Meza, S. (1997). La cuestión ganadera y la deforestación. México: . Universidad de Artes y Ciencias del Estado de Chiapas, Centro de Estudios Superiores de México Centroamérica.

Zamora-Martinez, M.C., Velasco, B.E., González, H.A., y Hernández, G. G. (2011). Modelos predictivos para la producción de productos forestales no maderables: Palma Camedor. (INIFAP, Ed.) (Manual téc). México, D.F. 